\title{
A non-flagellated biocontrol bacterium employs a PilZ-PilB complex to provoke twitching motility associated with its predation behavior
}

\author{
Long Lin', Mimi Zhou', Danyu Shen', Sen Han', Alex M. Fulano', Shan-Ho Chou and Guoliang Qian ${ }^{1 *}$ (D)
}

\begin{abstract}
Lysobacter enzymogenes $\mathrm{OH} 11$ is a non-flagellated, ubiquitous soil bacterium with broad-spectrum antifungal activities. Although lacking flagella, it employs another type of motile behavior, known as twitching motility that is powered by type IV pilus (T4P) to move towards neighboring crop fungal pathogens to kill them as food. At present, little is known about how this non-flagellated bacterium controls twitching motility that is crucial for its predatory lifestyle. Herein, we present a report on how a non-canonical PilZ domain, PilZ Le3639, controls such motility in the non-flagellated L. enzymogenes; it failed to bind with c-di-GMP but seemed to be required for twitching motility. Using bacterial two-hybrid and pull-down approaches, we identified PilB $B_{\text {Le0708, one of the }}$

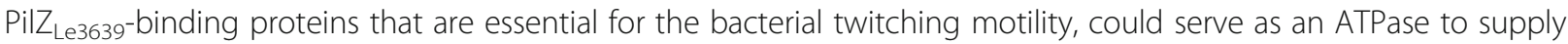
energy for T4P extension. Through site-mutagenesis approaches, we identified one essential residue of PilZ Le3639 $_{\text {en }}$ that is required for its binding affinity with PilB $\mathrm{B}_{\text {Le0708 }}$ and its regulatory function. Besides, two critical residues within

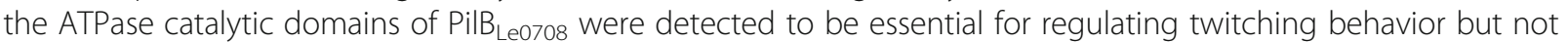

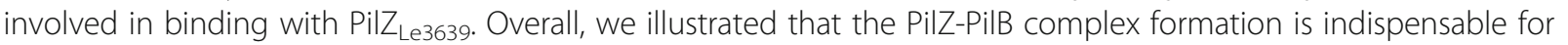
twitching motility in a non-flagellated bacterium.
\end{abstract}

Keywords: Twitching motility, PilZ, PilB, Lysobacter, Type IV pilus

\section{Background}

The Gram-negative genus of Lysobacter comprises more than 30 species that are emerging as important sources of crop biocontrol agents. They are novel because they exhibit proficient abilities to generate a wide variety of anti-infectious metabolites and extracellular lytic enzymes (Christensen and Cook 1978; Kobayashi et al. 2005; Xie et al. 2012; Panthee et al. 2016). Recent comparative genomic studies uncovered that most members of Lysobacter do not carry a FliC homolog, which encodes flagellin

\footnotetext{
* Correspondence: glqian@njau.edu.cn

'Key Laboratory of Integrated Management of Crop Diseases and Pests, College of Plant Protection, Laboratory of Plant Immunity, Nanjing Agricultural University, No.1 Weigang, Nanjing City 210095, Jiangsu Province, China

Full list of author information is available at the end of the article
}

required for the biogenesis of surface-attached flagella (de Bruijn et al. 2015). Therefore, with minor exceptions, most Lysobacter species are, although ubiquitous in the environment, non-flagellated (Christensen and Cook 1978; Hayward et al. 2010).

How do the Lysobacter species without flagella migrate towards a more favorable environment or escape from undesirable conditions in their natural niches? Our earlier studies revealed that surfaced-attached type IV pilus (T4P) can power Lysobacter species to move in a twitching mode (Zhou et al. 2015). This motile behavior seems to facilitate the non-flagellated Lysobacter species to draw near ecologically-relevant pathogens. When Lysobacter species establish the contact with pathogens, they

(c) The Author(s). 2020 Open Access This article is licensed under a Creative Commons Attribution 4.0 International License, which permits use, sharing, adaptation, distribution and reproduction in any medium or format, as long as you give appropriate credit to the original author(s) and the source, provide a link to the Creative Commons licence, and indicate if changes were made. The images or other third party material in this article are included in the article's Creative Commons licence, unless indicated otherwise in a credit line to the material. If material is not included in the article's Creative Commons licence and your intended use is not permitted by statutory regulation or exceeds the permitted use, you will need to obtain permission directly from the copyright holder. To view a copy of this licence, visit http://creativecommons.org/licenses/by/4.0/ 
predate or kill them, hence protect the plants from being infected (Xia et al. 2018).

L. enzymogenes is a plant-associated, soil proteobacterium that acts as a predator of crop fungal pathogens (Qian et al. 2009). To prey on nearby fungi, L. enzymogenes is required to move towards them via T4P-dependent twitching motility and further kills these microorganisms through secreting an antibiotic, known as heat-stable antifungal factor, HSAF (Yu et al. 2007; Xia et al. 2018). In our earlier studies, we demonstrated 19 pilus structural or regulatory component proteins, including the major pilus subunit, PilA and the motor proteins PilB and PilT, are required for the biogenesis of T4P and the function of twitching motility in $L$. enzymogenes OH11 (Xia et al. 2018).

Genes encoding proteins with PilZ domains are widely distributed in a variety of bacterial genomes and control numerous cellular processes, such as biofilm formation, T4P biogenesis, twitching motility, two-component signal transduction, and virulence (Galperin and Chou 2020). These domains could be stand-alone, in tandem, or fused with other signaling domains, such as EAL, GGDEF, or HD-GYP that are highly associated with the signaling of cdi-GMP, a ubiquitous bacterial second messenger Römling et al. 2013, 2005). The first defined PilZ domain is PilZ ${ }_{\mathrm{PA} 2960}$ that was found in Pseudomonas aeruginosa, a human pathogen and model flagellated bacterium (Alm et al. 1996). The PilZ $Z_{\text {PA2960 }}$ protein has only one domain, PilZ (Alm et al. 1996; Guzzo et al. 2009), which controls T4P formation and twitching behavior. PilZ ${ }_{\text {PA2960 }}$ regulates the secretion of PilA polymers to the cell surface (Alm et al. 1996). At the mechanistic level, while PilZ ${ }_{\text {PA2960 }}$ appears to regulate the T4P formation via protein-protein interactions, the PilZ $\mathrm{PA2960}_{\mathrm{P}}$-binding partners are yet to be characterized

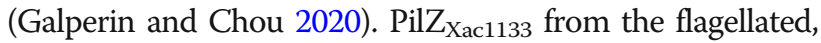
pathogenic Xanthomonas citri is homologous to the PilZ $Z_{\text {PA2960 }}$ protein. Crystal structural analyses revealed that PilZ ${ }_{\text {Xac1133, like PilZ }}$ PA2960, lacks two conserved signature motifs, RxxxR and $[\mathrm{D} / \mathrm{N}] \mathrm{xSxxG}$, that are required for c-diGMP binding (Guzzo et al. 2009; Li et al. 2009, 2011). PilZXac1133 not only binds with PilB Xac3239 $_{3}$ but also with the degenerated EAL domain of FimX $\mathrm{X}_{\mathrm{Xac} 398}$. FimX $\mathrm{X}_{\mathrm{PA} 4959}$ protein of $P$. aeruginosa, a homolog of Fim $\mathrm{X}_{\mathrm{Xac} 398}$, has also been found to be an essential regulator of T4P formation and twitching motility (Huang et al. 2003; Guzzo et al. 2009). The discovery of these various protein-protein interactions implies that $\mathrm{PilZ}_{\mathrm{Xac1133}}$ is involved in regulating T4P formation; however, no corresponding phenotypic evidence is available. Moreover, PilZ ${ }_{\text {Xac1133, }}$ unlike PilZ ${ }_{\text {PA2960, }}$ did not seem to control twitching motility as described previously (Guzzo et al. 2009). In agreement, X. campestris PliZ $Z_{\mathrm{Xc1028}}$, which is almost identical to $\mathrm{PilZ}_{\mathrm{Xac1133}}$, was found to play only a minor role in the twitching behavior (McCarthy et al. 2008). A similar situation has also been observed in the non-flagellated Neisseria meningitides
(Carbonnelle et al. 2005). Therefore, PilZ $\mathrm{PA} 2960$ and its homologs appear to play diversified roles in modulating the formation of T4P and/or twitching motility in different bacterial species with and without flagella.

A search of the genome of strain OH11 led us to discover Le3639, a homologous PilZ ${ }_{\text {PA2960 }}$ protein that is referred to as PilZ $Z_{\mathrm{Le} 3639}$ herein. PilZ $\mathrm{Le3639}_{\text {shares a very high }(76-83 \%)}$ sequence similarity with PilZ $_{\mathrm{PA} 2960}$ and contains no conserved c-di-GMP-binding signature motifs, as described above (Fig. 1a). The objective of this work is to determine whether PilZ $\mathrm{Le3639}_{\mathrm{L}}$ can affect the twitching motility of the non-flagellated $L$. enzymogenes, and if yes, what is the underlying mechanism? Herein, we showed that PilZ ${ }_{\text {Le3639 }}$ is indeed involved in controlling the twitching motility of $L$. enzymogenes. We further found that $\mathrm{PilZ}_{\mathrm{Le} 3639}$ directly interacted with PilB $\mathrm{Le}_{\mathrm{Le} 008}$, which is essential for twitching motility in L. enzymogenes. Moreover, PilZ $\mathrm{Le}_{\text {Le39 }}$ seems to use the same residue critical for its role in twitching motility and interaction with $\mathrm{PilB}_{\mathrm{Le} 0708}$. We underscore for the first time the involvement of the PilZ-PilB protein complex in twitching motility in non-flagellated bacteria, which provides further insights on the functional and mechanistic diversity of PilZ domains in bacteria.

\section{Results

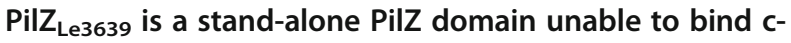 di-GMP}

A genomic search of strain $\mathrm{OH} 11$ by using PilZ $\mathrm{PA2960}_{\text {as }}$ the bait enabled us to identify PilZ $\mathrm{Le}_{\text {63639, a homologous }}$ protein in L. enzymogenes with a stand-alone PilZ domain (Fig. 1a) that shared 76\%, 82\%, and 83\% sequence similarity with PilZ ${ }_{\mathrm{PA} 2960}, \mathrm{P}_{1} Z_{\mathrm{Xac1133}}$, and PilZ $\mathrm{X}_{\mathrm{Xc1028}}$, respectively. The homologous $\mathrm{PilZ}_{\mathrm{Le3639}}$ proteins were also present in several selected Lysobacter species (Fig. 1a). Multiple sequence alignment revealed that $\mathrm{PilZ}_{\mathrm{Le} 3639}$ and its homologs from other selected Lysobacter species all lacked the conserved c-di-GMP-binding motifs described above (Fig. 1a). To experimentally validate this finding, we purified the $\mathrm{PilZ}_{\mathrm{Le} 3639}$-His fusion protein and subjected it to the microscale thermophoresis (MST) assay. We found no binding signals between the c-di-GMP

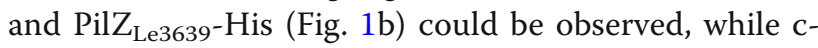
di-GMP bound well to the control protein Clp (with a $\mathrm{K}_{\mathrm{d}}$ of $0.21 \mu \mathrm{M}$ ), a known c-di-GMP-binding transcription factor according to our earlier work (Fig. 1c; Xu et al. 2018). These results suggest that the non-flagellated Lysobacter species carry a conserved stand-alone PilZ domain that does not appear to interact with c-di-GMP as evidenced by PilZ $Z_{\text {Le3639. }}$.

\section{Pil $Z_{\text {Le3639 }}$ is essential for modulating the twitching motility of Lysobacter}

To investigate whether $\mathrm{PilZ}_{\mathrm{Le3639}}$ exhibits a role in affecting the Lysobacter twitching motility, we generated 

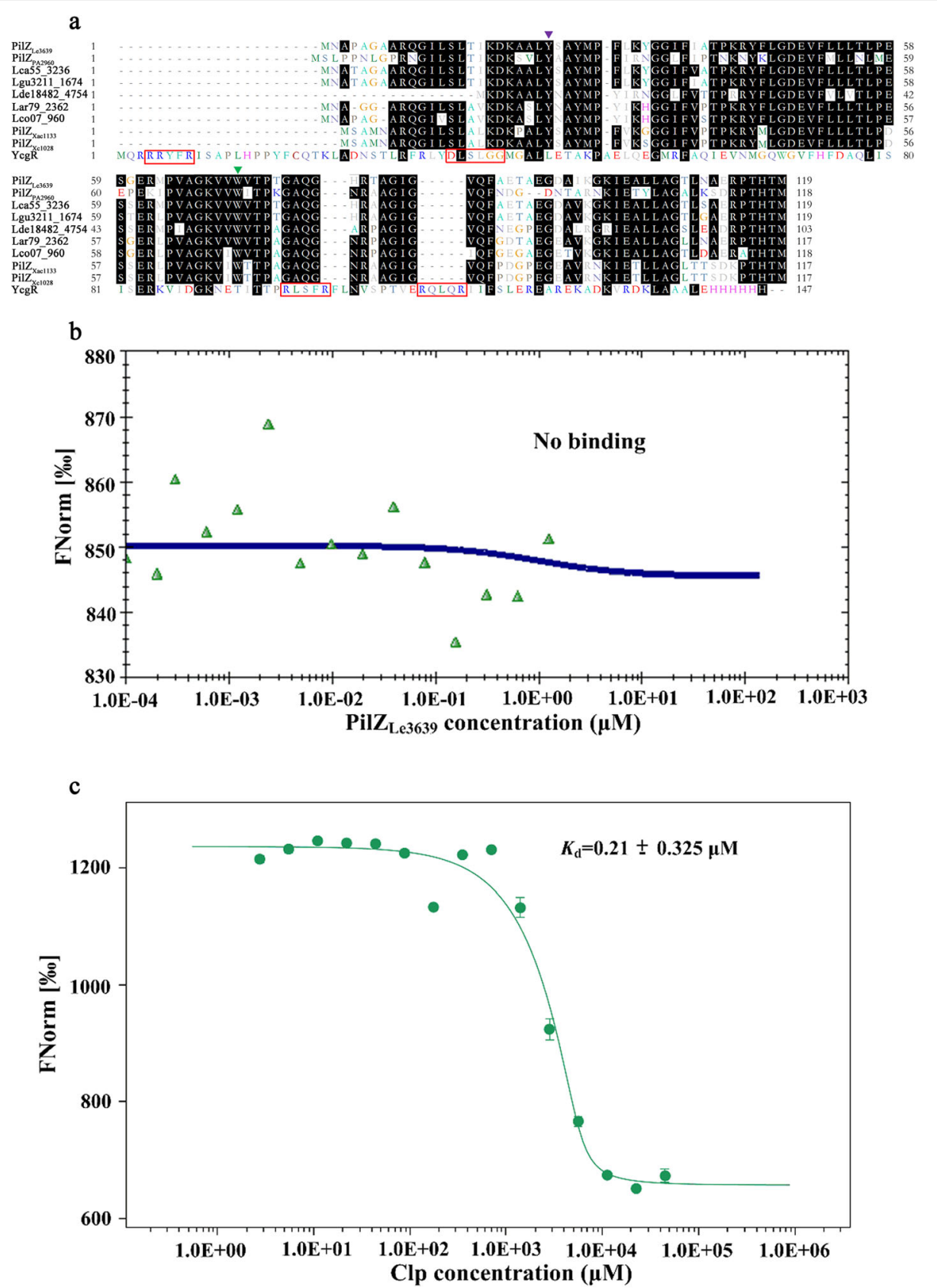

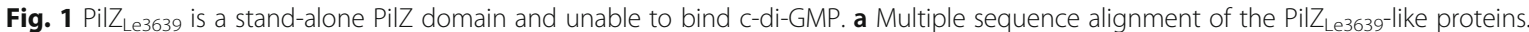
PilZLe3639, PilZpa2960, Lca55_3236, Lgu3211_1674, Lde18482_475, Lar79_2362, Lco07_960, PilZXac1133 and PilZ Xc1028 indicated these stand-alone PilZ domains from Lysobacter enzymogenes OH11, Pseudomonas aeruginosa PA01, L. capsisi 55, L. gumosus 3.2.11, L. defluvii DSM18482, L. arseniciresistens ZS79, L. concretionis Ko07, Xanthomonas citri and X. campestris, respectively. YCgR (PDB entry 5Y6G), is a c-di-GMP-binding protein from E. coli. The RxxxR and [D/N] xSxxG that are required for c-di-GMP binding (Guzzo et al. 2009) are highlighted by red boxes. Two conserved residues (Y24 and W71) in the selected stand-alone PilZ domains are indicated by purple and green triangles, respectively. b Microscale thermophoresis (MST) assay showed PilZ Le3639 failed to bind with c-di-GMP, while c Clp, a known c-di-GMP-binding protein (Xu et al. 2018), showed moderate binding affinities with c-di-GMP determined by MST

$\triangle$ PilZ $_{\text {Le3639, }}$ an in-frame Lysobacter deletion mutant (Additional file 1: Table S1) via a double homologousrecombination approach as described earlier (Qian et al. 2012). In the twitching-motility inducing medium $(1 / 10$ TSA), we found that the wild-type OH11 displayed an apparent twitching behavior by spreading single or clustered cells outside from the colony margin (Fig. 2). At the same time, the inactivation of PilZ $\mathrm{Le3639}$ completely abolished this twitching phenomenon (Fig. 2). The twitching motility defect in the $P i l Z_{L e 3639}$ mutant was 


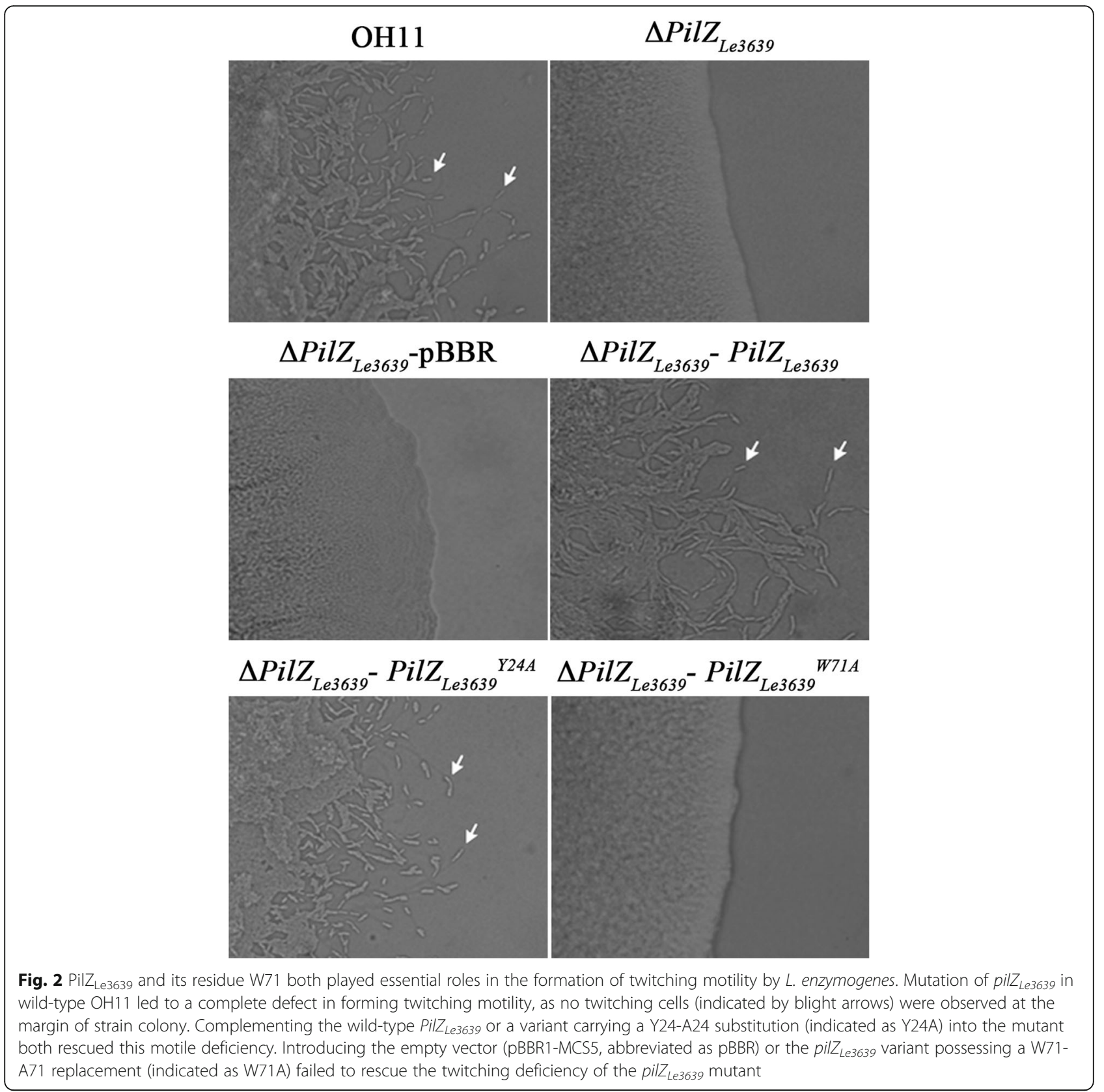

fully rescued by complementation with a plasmid-borne PilZ $_{\text {Le3639 }}$ (Fig. 2) but not by an empty plasmid (Fig. 2). These results imply the crucial role of $\mathrm{PilZ}_{\mathrm{Le} 3639}$ in triggering the Lysobacter twitching motility. According to an earlier study (Guzzo et al. 2009), Tyr24 (Y24) and Trp71 (W71) are the two potential key residues for executing the biological function of PilZ $\mathrm{Xac1133}_{\text {. To figure }}$ out whether they also play a similar role in PilZ Le3639, $_{\text {, }}$ we generated two individual mutants of $\mathrm{Y} 24 \mathrm{~A}$ ( $\mathrm{A}$ is an abbreviation of Alanine) and W71A, respectively, and complemented these PilZ $_{L e 3639}$ variants with the plasmid harboring Y24A or W71A substitution. The results (Fig. 2) showed that the Y24 mutant behaves like the wild- type allele and can rescue the twitching motility defect of the pilZ $Z_{\text {Le3639 }}$ mutant. However, the W71 mutant allele did not restore the normal twitching motility phenotype, indicating W71 is a key residue for protein activity. These results collectively suggest that $\mathrm{PilZ}_{\mathrm{Le} 3639}$ is required for twitching motility, and the amino residue of W71 is essential for displaying such a function.

\section{Binding of PilZ $\mathrm{Le3639}_{\text {with }} \mathrm{PilB}_{\mathrm{Le0708}}$ is required for twitching motility}

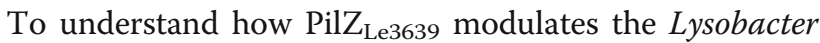
twitching motility, we screened a total of 19 pilusassociated structural or regulatory proteins (Xia et al. 
2018) to identify the direct PilZ $\mathrm{Le3639}_{\text {-binding partner(s) }}$ (Fig. 3a). For screening, we employed the bacterial adenylate cyclase two-hybrid (BACTH) system (Ouellette et al. 2017). Each of the 19 genes was individually cloned into the pKT25 vector to generate the prey pKT25 constructs. Along with the bait plasmid pUT18c-PilZ $\mathrm{Le3639}_{\text {, }}$ each of the recombinant prey pKT25 constructs was cotransformed into the E. coli BTH101, which was further grown in a selective medium. Based on the principle of the BACTH system, if bait PilZ $\mathrm{Le}_{\mathrm{L} 639}$ established a direct interaction with the prey protein, the co-transformed $E$. coli strain would exhibit a "blue" phenotype. Via this approach, we found that among the 19 proteins, only $\mathrm{PilB}_{\mathrm{Le} 0708}$ interacted directly with PilZ $\mathrm{Le}_{\text {L639 }}$ (Fig. 3b). To validate this observation, we further tested the ability of PilZ $\mathrm{Le3639}_{\text {-His }}$ to pull down GST-PilB ${ }_{\mathrm{Le} 0708}$, which was indeed the case since we observed a positive signal (Fig. 3c). These results reveal that PilZ $\mathrm{Le3639}$ directly interacted with PilB $_{\text {Le0708 }}$ of L. enzymogenes.

Multiple sequence alignment showed that $\mathrm{PilB}_{\mathrm{Le} 0708}$ possessed two conserved motifs, the Walker A and Walker B (boxed in red rectangular in Fig. 4a), both of which are highly required for its enzymatic activity (Chiang et al. 2008). Between the predicted Walker A and B motifs, the residues of Lys333 (K333) and Glu397 (E397) of PilB Xac3239 were previously shown to play potential key roles in forming the PilB $\mathrm{Xac3239}-\mathrm{PilZ}_{\mathrm{Xac1133}}$ complex (Guzzo et al. 2009). Therefore, we constructed the $\mathrm{PilB}_{\mathrm{Le} 0708}$ variants with a K333A or E397A substitution in the wild-type chromosome and subsequently tested the ability of the mutant strains in producing the $L$. enzymogenes twitching motility. We found that the chromosomal replacement of Lys333 or Glu397 in the wild-type $\mathrm{OH} 11$ completely abolished the bacterial twitching motility (Fig. 4b), suggesting that both residues are essential for the function of $\mathrm{PilB}_{\mathrm{Le} 0708}$, which supports the earlier notion that the enzymatic activity and functionality of PilB are highly associated. To validate that the above observations are site specific, two unrelated residues, Pro208 or Tyr209 of $\mathrm{PilB}_{\mathrm{Le} 0708}$, were further selected for replacement by Alanine (A) (Fig. 4a). We found that the P208A or Y209A substitution did not seem to affect the $L$. enzymogenes twitching motility (Fig. 4b). Therefore, the PilZ ${ }_{\mathrm{Le} 3639}-\mathrm{PilB}_{\mathrm{Le} 0708}$ binding pair appears to correlate well with their functional outcomes in regulating the formation of twitching motility in L. enzymogenes.

\section{W71 of PilZ $\mathrm{Le3639}_{\text {is }}$ required for PilZ $\mathrm{Le3639}_{\text {-PilB }}$ Le0708 interaction}

To provide molecular details for the PilZ $\mathrm{Le3639}_{\mathrm{L}}-\mathrm{PilB}_{\mathrm{Le} 0708}$ binding, we tried to identify key residue(s) of PilZ $Z_{\mathrm{Le} 3639}$ or
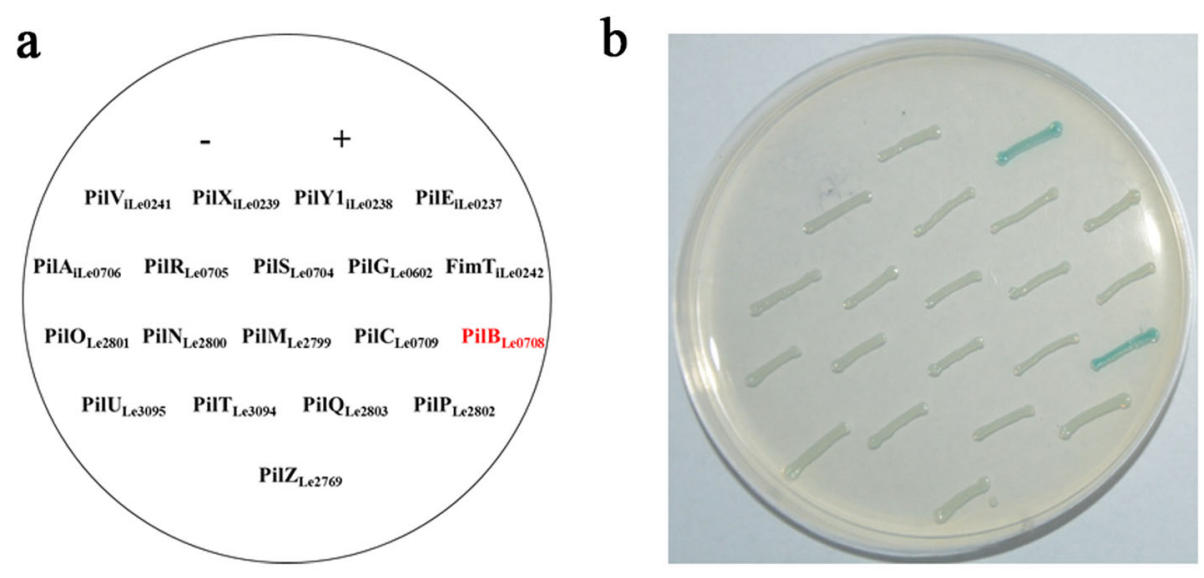

c

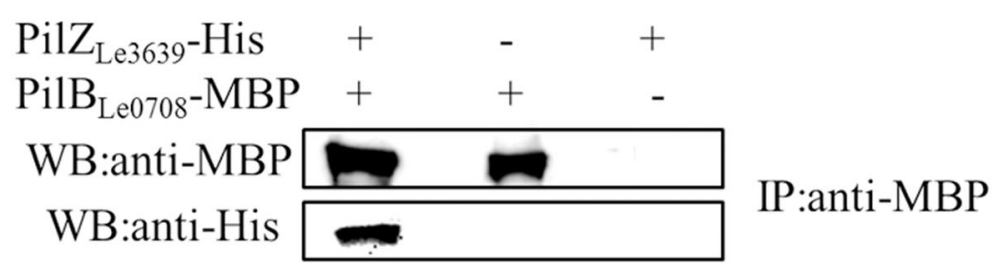

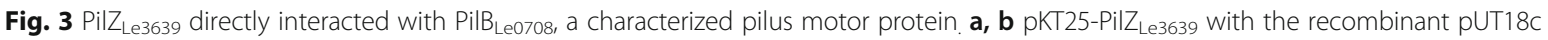
carrying different pilus-associated proteins that are required for twitching motility in L. enzymogenes OH11 (Xia et al. 2018 ) were co-transformed into the E. coli BTH101 cells to test their potential protein-protein interactions by the bacterial adenylate cyclase two-hybrid (BACTH) system. The co-transformed E. coli colony with a "blue" color indicated the test protein-protein interactions happened under the test conditions. Using this

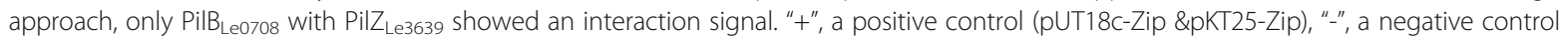
(pUT18c \&pKT25). c GST pull-down assay confirmed the direct binding of PilZ $Z_{\text {Le3639 }}$ with PilB Le0708 $_{\text {in vitro }}$ 
a

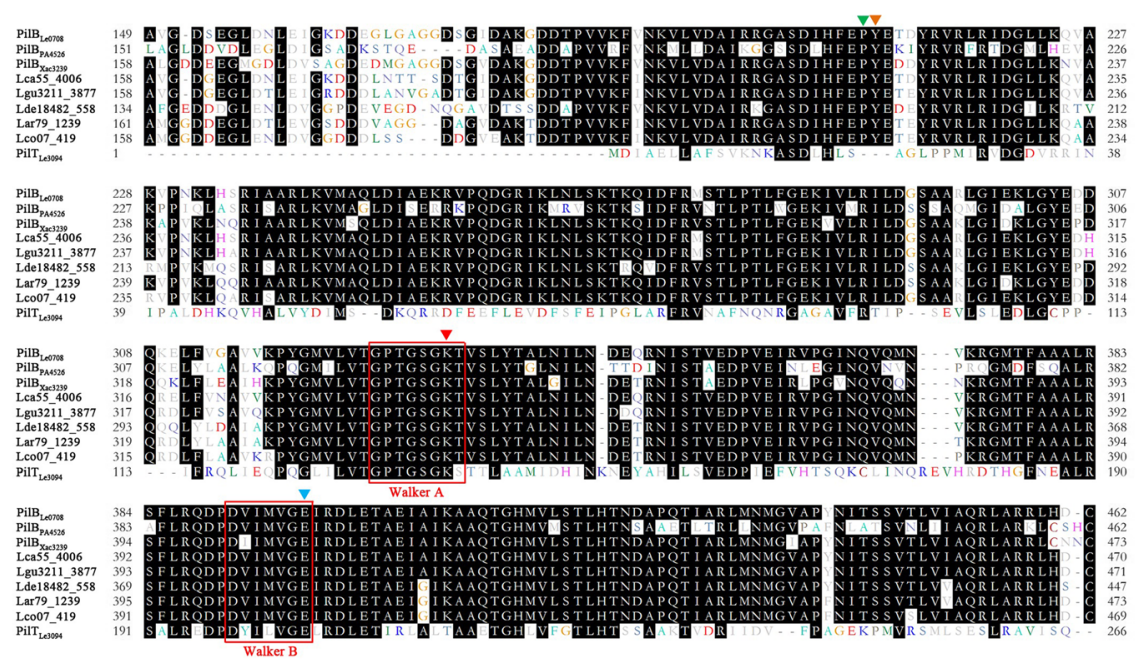

b

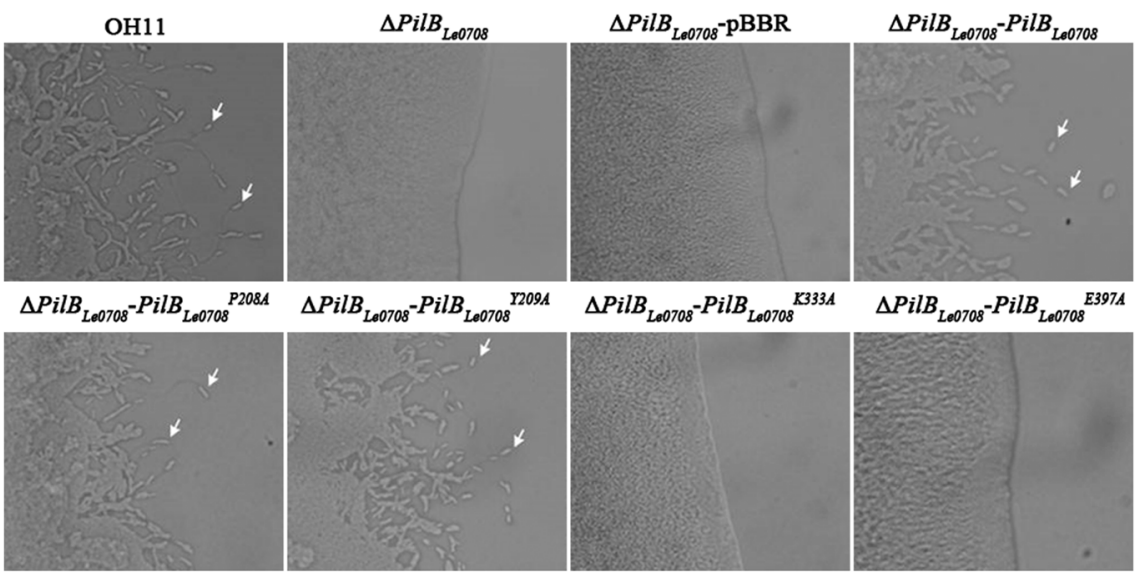

Fig. 4 The K333 and E397 residues were required for the regulation of PilB $B_{L e 0708}$ in the formation of twitching motility in L. enzymogenes OH11. a

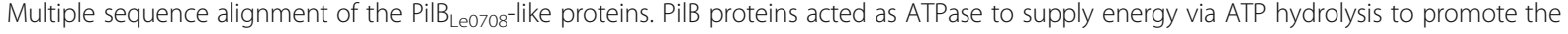

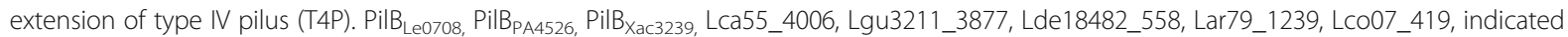
these PilB proteins from Lysobacter enzymogenes OH11, Pseudomonas aeruginosa PA01, Xanthomonas citri, L. capsisi 55, L. gumosus 3.2.11, L. defluvii DSM18482, L. arseniciresistens ZS79, and L. concretionis Ko07, respectively. PilTLe3094 served as the PilT protein from L. enzymogenes OH11, which functioned as ATPase to supply energy via ATP hydrolysis to elicit the retraction of T4P. "Walker A" and "Walker B" are two known motifs that are essential for the ATPase activity of the PilB or PilT family proteins. The conserved P208, Y209, K333 and E397 residues are indicated by green, orange, red and blue triangles, respectively. $\mathbf{b}$ Involvement of the K333 and E397 residues in the regulation of PilB Le0708 $_{\text {in }}$ twitching formation. Mutation of PilB $B_{L e 0708}$ in wild-type OH11 fully impaired twitching motility, while complementation of the plasmid-borne pilB $B_{\text {Le0708 }}$ rescued this defect. At the wild-type background of strain OH11, chromosomal replacement of K333 to A333 (K333A) or E397 to A397 (E397A), but not P208A208 (P208A) and Y209-A209 (Y209A) abolished the strain in producing twitching cells

PilB $_{\mathrm{Le} 0708}$ that are important for their binding and function. For this purpose, we first selected the residues of

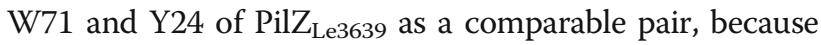
we already showed that the former was necessary for the involvement of PilZ $\mathrm{Le3639}_{\text {in }}$ in twitching motility, while the latter was not (Fig. 2). Using the BACTH system, we

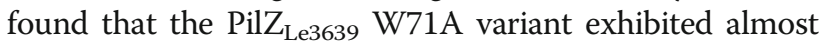
entirely impaired binding capability, while the PilZ $\mathrm{Le3639}$ Y24A variant did not seem to demonstrate any binding with $\mathrm{PilB}_{\mathrm{Le} 0708}$ (Fig. 5a). These results collectively indicate that W71 is a key residue of PilZ $\mathrm{Le3639}_{\text {in }}$ in determining its binding affinity with $\mathrm{PilB}_{\mathrm{Le} 0708}$ and to regulate the in vivo twitching motility.

To map the residues of $\mathrm{PilB}_{\mathrm{Le} 0708}$ that are essential for influencing its direct interaction with $\mathrm{PilZ}_{\mathrm{Le} 3639}$, we tested the binding of the PilZ $\mathrm{Le3639}_{\text {}}$ with several Pil$\mathrm{B}_{\mathrm{Le} 0708}$ variants $\left(\mathrm{PilB}_{\mathrm{Le} 0708}{ }^{\mathrm{K} 333 \mathrm{~A}}\right.$, PilB ${ }_{\mathrm{Le} 0708}{ }^{\mathrm{E} 337 \mathrm{~A}}$, Pil$\mathrm{B}_{\mathrm{Le} 0708}{ }^{\mathrm{P} 208 \mathrm{~A}}$, and $\mathrm{PilB}_{\mathrm{Le} 0708}{ }^{\mathrm{Y} 09 \mathrm{~A}}$ ) through the BACTH system and found that, like the native $\mathrm{PilB}_{\mathrm{Le} 0708}$, all the tested $\mathrm{PilB}_{\mathrm{Le} 0708}$ variants seemed to be able to bind well with PilZ $\mathrm{Le3639}_{\mathrm{L} 3}$ under the testing conditions (Fig. 5b). These results suggest that while K333 and E397 of 
a

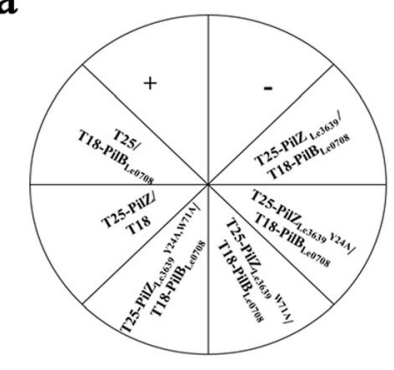

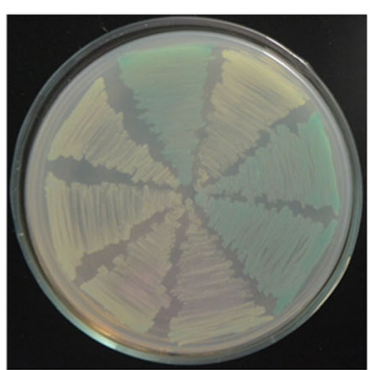

C

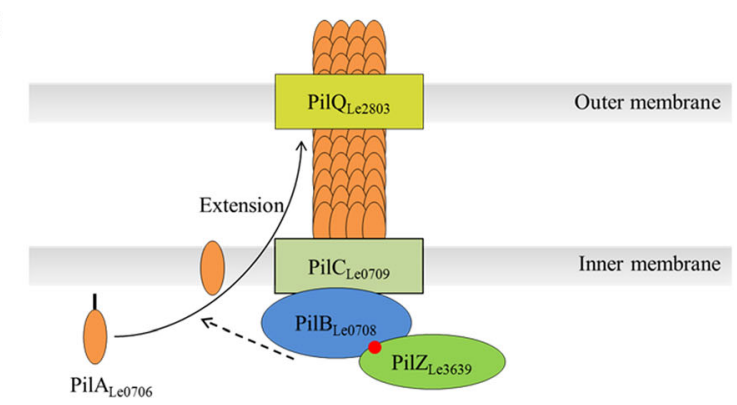

b
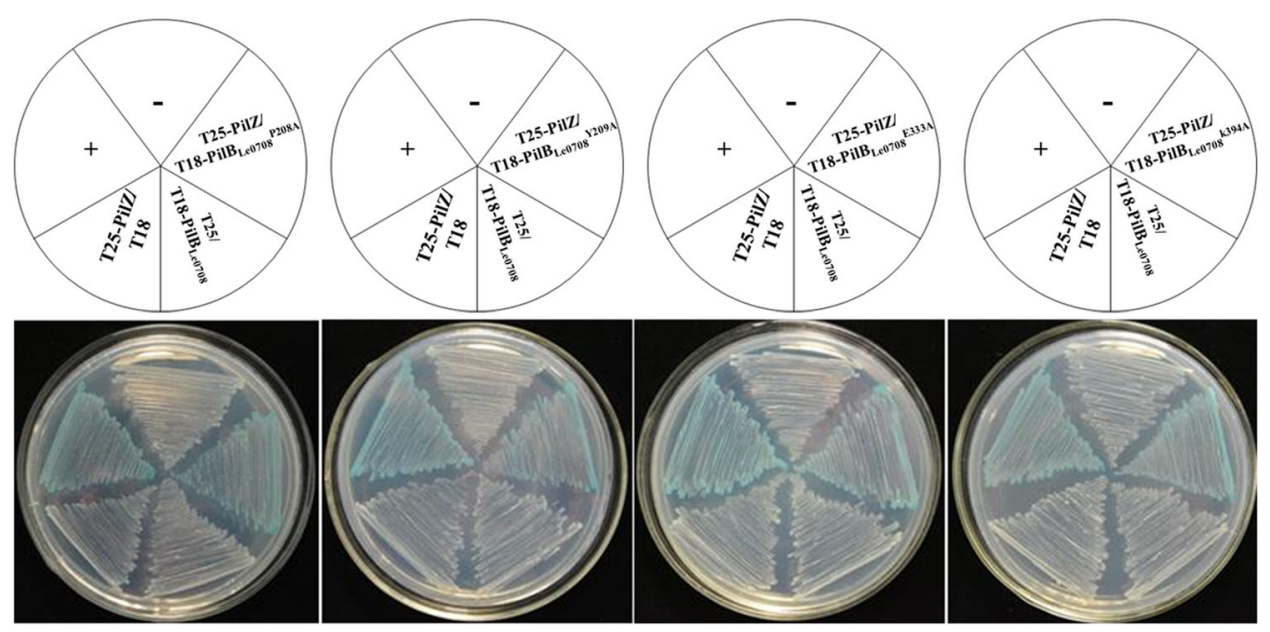

Fig. 5 Identification of key amino residues of PilZ $\mathrm{Le3639}_{9}$ or PilB $\mathrm{L}_{\mathrm{Le} 0708}$ that required for their binding determined by the BACTH system. a The W71,

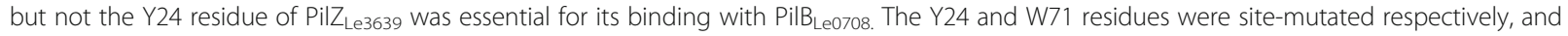

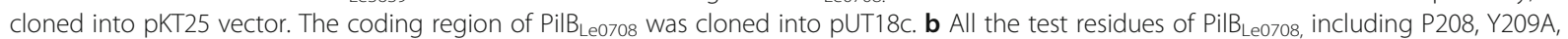

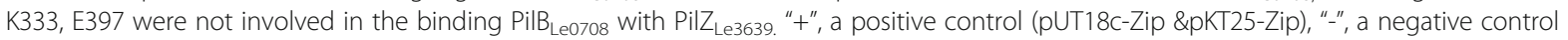
(pUT18c \&pKT25). c A schematic model for the PilZ Le3639-PilB Le0708 complex regulating T4P-dependent twitching motility in Lysobacter enzymogenes $\mathrm{OH} 11$. PilB $\mathrm{B}_{\mathrm{Le} 008}$ is an ATPase responsible for pilus extension. PilZ Le3639 may activate (indicated by a dashed arrow) pilus extension

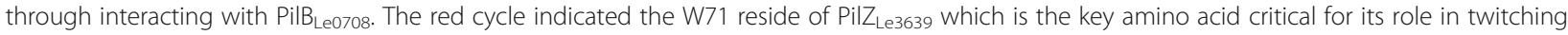
motility and interaction with $\mathrm{PilB}_{\mathrm{Le0708}}$ PilC $_{\mathrm{Le0709}}$ is an integral membrane protein functioning as the platform protein of pilus, while $\mathrm{PilQ}_{\mathrm{Le} 2803}$ acts as the outer membrane secretin pore (Xia et al. 2018)

$\mathrm{PilB}_{\mathrm{Le0708}}$ are required for its own regulation in twitching motility, both of them are not likely involved in the $\mathrm{PilB}_{\mathrm{Le} 0708}-\mathrm{PilZ}_{\mathrm{Le} 3639}$ binding.

\section{Discussion}

Twitching motility is arguably one of the bestcharacterized motile behaviors in bacteria (Burrows 2012). Unlike the flagellum-driven swimming or swarming motility, the formation of twitching motility is powered instead by the type IV pilus (T4P) (Chang et al. 2016). Not only for motility, the plant and human pathogenic bacteria also use T4P-mediated twitching motility to promote bacterial infections (Burrows 2012; Dunger et al. 2016; Corral et al. 2020). In the past decade, the transcription, posttranscription, and post-translation regulations of twitching motility have been well-documented from various bacterial systems (Burrows 2012; Craig et al. 2019). Among the twitching motility regulators, the PilZ $\mathrm{PA} 2960$ protein is an original, stand-alone PilZ domain that exhibits both post- translation and protein-protein interaction capabilities in controlling T4P biogenesis and twitching motility (Alm et al. 1996; Guzzo et al. 2009). Afterwards, several PilZ $Z_{\mathrm{PA} 2960}$ homologs, i.e. PilZ $\mathrm{Xac1133}_{\text {and }}$ PilZ $\mathrm{X}_{\mathrm{Xc1028}}$, were identified from the flagellated, plant pathogenic Xanthomonas spp. (Guzzo et al. 2009). Interestingly, unlike PilZ ${ }_{\mathrm{PA} 2960}$, the PilZ $\mathrm{Xac133}_{\mathrm{X} 13}$ and PilZ $\mathrm{X}_{\mathrm{Xc1028}}$ proteins are potentially not involved in the establishment of twitching motility in their respective host systems (McCarthy et al. 2008; Guzzo et al. 2009). These earlier observations indicate the diversified roles of the homologous PilZ $\mathrm{PA} 2960_{\text {proteins in }}$ different bacterial species with flagella. In the previous study, we provided solid evidence to show that PilZ $\mathrm{Le3639}_{\text {is }}$ indeed a homolog of PilZ $\mathrm{PA2960}_{\text {, }}$ and was able to regulate twitching motility in the non-flagellated soil bacterium of L. enzymogenes (Qian et al. 2009). In this aspect, PilZ $\mathrm{L}_{\mathrm{Le} 3639}$ seems to be involved in twitching motility prompting nonflagellated bacterium to move towards fungi to a close range for contact (Patel et al. 2010, 2011). The resulting 
interaction of $L$. enzymogenes-fungi favours the nonflagellated bacteria to kill fungal pathogens to acquire nutrients, an aspect regarded as gaining adaptive advantage in natural niches. To our knowledge, this is the first report of the involvement of the widespread stand-alone PilZ domains in controlling twitching motility in non-flagellated bacteria, particularly in the biocontrol agent of phytopathogenic fungi.

In this study, we provide some further insights into how the stand-alone PilZ domains affect twitching motility in non-flagellated bacteria. In our case (Fig. 5c), we found that PilZ $\mathrm{L}_{\mathrm{Le} 3639}$ formed a binary complex with Pil$\mathrm{B}_{\mathrm{Le} 0708}$, which is needed to transduce the signaling event for twitching motility. While the PilZ ${ }_{\text {Xac1133}}-$ PilB $_{\text {Xac3239 }}$ binding has been proved under the in vitro condition in the flagellated Xanthomonas spp. (Guzzo et al. 2009), whether such a protein-protein interaction contributes to twitching motility regulation is, however, not experimentally documented. Moreover, besides PilB Xac3239, PilZ Xac1133 also directly interacts with FimX $X_{\text {Xac2398, }}$, which is a known regulator of T4P (Guzzo et al. 2009). Again, direct experimental evidence supporting the involvement of the PilZ $\mathrm{Xac1133}$-FimX $\mathrm{X}_{\mathrm{Xac} 2398}$ complex in twitching motility is also lacking. Despite the hypothesis that PilZ ${ }_{\text {PA2960 }}$ most likely forms a complex with a T4P regulator and/or structural-component to co-activate the production of twitching motility in $P$. aeruginosa, both the PilZ $\mathrm{PA} 2960-\mathrm{PilB}_{\mathrm{PA} 4526}$ and PilZ $_{\mathrm{PA} 2960}$-FimX $\mathrm{X}_{\mathrm{PA} 4959}$ bindings, however, were not observed in this bacterium (Qi et al. 2012). Instead, FimX $X_{\text {PA4959 }}$ was shown to form a complex directly with PilB $\mathrm{PA} 4526_{2}$ in $P$. aeruginosa, and this complex is vital for the "correct" polar-localization of both partners, which is essential for their coinvolvement in the assembly of T4P (Jain et al. 2017). Therefore, the discovery of the PilZ $\mathrm{Le3639}-\mathrm{PilB}_{\mathrm{Le} 0708}$ binding presented in this study represents the first evidence that PilZ-PilB complex formation is indispensable for bacterial twitching motility. Besides, we observed that PilZ $Z_{\text {Le3639 }}$ uses a crucial residue of W71 to bind with $\mathrm{PilB}_{\mathrm{Le} 0708}$ and regulate bacterial twitching behavior, which provides a strong association between the $\mathrm{PilZ}_{\mathrm{Le} 3639}-\mathrm{PilB}_{\mathrm{Le} 0708}$ binding and their co-regulation on twitching motility. What is the potent advantage for $L$. enzymogenes to form the PilZ $\mathrm{Le}_{\mathrm{L} 639}-\mathrm{PilB}_{\mathrm{Le} 0708}$ complex? Our earlier works showed that $L$. enzymogenes indeed stimulated its own twitching motility when a nearby fungal pathogen is present but failed to generate twitching behavior under the nutrient-rich conditions (Zhou et al. 2015; Zhao et al. 2017). Based on these considerations, it is possible that upon sensing yet unidentified environmental or cellular stimuli, the non-flagellated L. enzymogenes becomes proficient in twitching motility. This attribute allows the biocontrol agent of crop fungal pathogens to access nutrients. It follows then that $L$. enzymogenes forms or disassembles the $\mathrm{PilZ}_{\mathrm{Le} 3639}$-Pil$\mathrm{B}_{\mathrm{Le} 0708}$ complex to move via twitching or to stop its motility when it lives in a nutrient-limited environment/ presence of nearby fungi or nutrient-rich environment/ absence of fungi, respectively. Such capacities could match the benefits and economic costs of $L$. enzymogenes in natural niches.

It is also noteworthy that the PilZ $\mathrm{Le}_{\mathrm{Le} 639}$ seems to interact with $\mathrm{PilB}_{\mathrm{Le} 0708}$, but not $\mathrm{PilT}_{\mathrm{Le} 3094}$, a protein sharing $37 \%$ sequence similarity with $\mathrm{PilB}_{\mathrm{Le} 0708}$ (Fig. 3b). At present, it is well recognized that PilB and PilT both function as ATPase, with the former supplying energy via ATP hydrolysis to promote T4P extension, while the latter inducing the retraction of T4P (Chiang et al. 2008). Therefore, the PilZ $\mathrm{Le3639}-\mathrm{PilB}_{\mathrm{Le} 0708}$ binding might support T4P extension, resulting in regulation of twitching motility. Besides, it was previously documented that

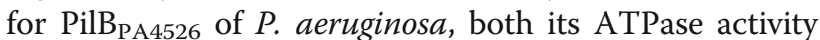
and polar localization in cells (Jain et al. 2017) are essential for its function. Whether PilZ $\mathrm{Le3639}$ regulates twitching motility by binding with $\mathrm{PilB}_{\mathrm{Le} 0708}$ to alter its ATPase activity and/or changing the $\mathrm{PilB}_{\mathrm{Le} 0708}$ cellular localization remains unknown.

It is also noteworthy that recent studies showed that several transcription regulators or chemical signaling systems controlled the T4P-driven twitching motility in L. enzymogenes through a direct or indirect transcription regulation of genes or gene clusters responsible for pilus biogenesis (Chen et al. 2017, 2018; Feng et al. 2019). Unlike these cases, the present study uncovered a protein complex formed by PilZ $\mathrm{Le3639}_{\text {and }}$ ailB $\mathrm{Pe}_{\mathrm{Le} 708}$ that seems to affect pilus extension and hence play a key role in the formation of twitching motility in L. enzymogenes. These findings collectively suggest that the non-flagellated soil bacterium, L. enzymogenes has similarly designed multiple molecular strategies to modulate the twitching behavior in response to environmental stimuli.

Finally, according to earlier studies, T4P-mediated twitching motility enables $L$. enzymogenes to fully exhibit antagonistic capability against crop pathogens (Xia et al. 2018). The motile behavior helps $L$. enzymogenes to predate on fungi to derive nutrients and to colonize on the plant/soil surface and/or fungal mycelium. In this viewpoint, the fundamental insights presented in this study might be helpful to increase the biocontrol efficiency of L. enzymogenes in crop field by promoting twitching motility through engineering a more stable PilZ-PilB complex formation.

\section{Conclusions}

While the mechanisms by which flagellated bacteria control the formation of type IV pilus and/or twitching motility have been studied extensively, those in non-flagellated bacteria remain mostly unknown. In the present study, we showed the 
mechanism by discovering the PilZ $\mathrm{Le3639}-\mathrm{PilB} \mathrm{B}_{\mathrm{Le} 0708}$ complex formation required for twitching motility in the nonflagellated, biocontrol bacterium, L. enzymogenes. The binding between PilZ $\mathrm{Le3639}_{\mathrm{L}}$ and the pilus-extension related motor protein $\mathrm{PilB}_{\mathrm{Le} 0708}$ seems to be specific, as PilZ $\mathrm{Le3639}_{\text {failed to }}$ interact with other pilus-retraction associated motor protein, PilT $_{\text {Le3094 }}$ that shares $37 \%$ sequence similarity with Pil$\mathrm{B}_{\mathrm{Le} 0708 \text {. These findings led us to propose that the PilZ }} \mathrm{Le3639}^{-}$ $\mathrm{PilB}_{\mathrm{Le} 0708}$ binding seems to influence the process of T4P extension in a non-flagellated, soil bacterium. Our findings not only expand our current knowledge on the mechanistic actions about how bacteria without flagella control the flagella-independent twitching behavior, but also reveal that the biocontrol $L$. enzymogenes likely engineers the PilZ-PilB complex to enhance twitching capacity. With such mechanism $L$. enzymogenes is able to search and predate on nearby fungal pathogens, leading to crop protection.

\section{Methods}

\section{Bacterial strains, plasmids and growth conditions}

The bacterial strains and plasmids used in the present study are listed in the Additional file 1: Table S1. The Escherichia coli strains that are used for plasmid construction were grown in Luria-Bertani (LB) medium with appropriate antibiotics at $37^{\circ} \mathrm{C}$. L. enzymogenes strains were cultivated in LB medium or 1/10 Tryptic Soy Broth (TSB) at $28^{\circ} \mathrm{C}$. When required, appropriate antibiotics (kanamycin, $\mathrm{Km}$, and gentamicin, $\mathrm{Gm}$ ) were added into the media.

\section{Genetic methods}

A double-crossover homologous recombination approach was used to generate an in-frame deletion mutant of Pil $Z_{\text {Le3639, }}$, as described previously (Qian et al. 2012). The primers used in this assay are listed in the Additional file 1: Table S2. In brief, the corresponding primers were employed to amplify the flanking regions of the target gene and cloned into the suicide vector pEX18Gm (Additional file 1: Table S1). The final construct was transformed into the wild-type OH11 by electroporation. After that, the Lysobacter transformants were selected on LB plates supplemented with $\mathrm{Km}$ and $\mathrm{Gm}$. Positive colonies were grown in LB medium for $6 \mathrm{~h}$ without antibiotics and subsequently plated on LB agar carrying $10 \%(\mathrm{w} / \mathrm{v})$ sucrose and $\mathrm{Km}$ to select the doublecrossover colonies. The final in-frame deletions were verified by PCR using the primers listed in the Additional file 1: Table S2.

Plasmid-based complementation assay was carried out as described previously (Qian et al. 2013). Briefly, different primer pairs listed in the Additional file 1: Table S2 were used to amplify the DNA fragment containing the coding region and the predicted promoter of the target gene by PCR, and the target DNA fragment was subsequently cloned into the broad-host vector pBBR1MCS5 (Additional file 1: Table S1). The final constructed plasmid was transformed into the mutant by electroporation and verified by PCR.

Chromosomal complementation or residue replacement was generated based on the double-crossover homologous recombination, as described previously (Xu et al. 2018). In brief, different primer pairs (Additional file 1: Table S2) were used to amplify DNA fragments containing the coding region and the flanking regions of each gene with or without point mutations by PCR. The purified PCR products were cloned into the suicide vector pEX18Gm to create a target construct (Additional file 1: Table S1), followed by transformation into the wild-type $\mathrm{OH} 11$ or mutants by electroporation. The selection of positive colonies and PCR confirmation were similar to those described above.

\section{Twitching motility assay}

We investigated twitching phenotype according to our earlier studies (Zhou et al. 2015; Xia et al. 2018). In brief, the wild-type $\mathrm{OH} 11$ and its derivatives were inoculated at the edge of a sterilized coverslip containing 1/20 tryptic soy agar (TSA) with $1.8 \%$ agar. Following a $24 \mathrm{~h}$ incubation at $28^{\circ} \mathrm{C}$, the margin of the test bacterial colonies on the microscope slide was observed under a light microscope. The twitching motility of L. enzymogenes was designated as motile cells or cell clusters growing away from the original colony (Zhou et al. 2015).

\section{Protein expression and purification}

The coding region of PilZ $\mathrm{Le}_{\mathrm{L} 639}$ and $\mathrm{PilB}_{\mathrm{Le} 0708}$ was amplified by PCR with the primers listed in the Additional file 1 : Table S2 and cloned into plasmid pET30a or pMAL-p2x to generate the $\mathrm{PilZ}_{\mathrm{Le} 3639}-\mathrm{His}_{6}$ and $\mathrm{PilB}_{\mathrm{Le} 0708}-\mathrm{MBP}$ protein fusion, respectively (Additional file 1: Table S1). For protein expression and purification, each resulting vector was transformed into $E$. coli BL21 (DE3) (Additional file 1: Table S1). The resulting strains were cultivated in LB medium (with $25 \mu \mathrm{g} / \mathrm{mL} \mathrm{Km}$ ) overnight at $37^{\circ} \mathrm{C}$. Two $\mathrm{mL}$ of the overnight culture was transferred into $200 \mathrm{~mL}$ of fresh $\mathrm{LB}$ at $37^{\circ} \mathrm{C}$ and grown with shaking at $220 \mathrm{rpm}$, until reaching an $\mathrm{OD}_{600}$ of 0.5 . Isopropyl $\beta$-D-1-thiogalactopyranoside (IPTG, Sigma, USA) was added to a final concentration, $0.8 \mathrm{mM}$. The culture was incubated for an additional $4 \mathrm{~h}$ at $28^{\circ} \mathrm{C}$. The cells were collected by centrifugation $(12,500$ $\mathrm{rpm}$ ) at $4{ }^{\circ} \mathrm{C}$ and resuspended in $20 \mathrm{~mL}$ of phosphatebuffered saline (PBS) lysis buffer with $10 \mathrm{mM}$ protease inhibitor (PMSF, Sigma, USA). The resulting cells were lysed with 30-min sonications (Sonifier 250; Branson Digital Sonifier, Danbury, USA), and the crude cell extracts were centrifuged at $12,500 \mathrm{rpm}$ at $4{ }^{\circ} \mathrm{C}$ for $25 \mathrm{~min}$. Soluble proteins containing the PilZ $\mathrm{Le3639}-\mathrm{His}_{6}$ were collected and mixed with pre-equilibrated $\mathrm{Ni}^{2+}$ resin (GE Healthcare, Shanghai, China) for $1 \mathrm{~h}$ at $4{ }^{\circ} \mathrm{C}$. The $\mathrm{PilZ}_{\mathrm{Le} 3639}-\mathrm{His}_{6}$ were 
placed in a column and washed with the re-suspension buffer with $30 \mathrm{mM}$ imidazole. The purified proteins were finally eluted in $250 \mathrm{mM}$ imidazole. The supernatant (soluble proteins containing thePilB $\mathrm{L}_{\mathrm{Le} 0708}-\mathrm{MBP}$ ) was passed through $1 \mathrm{~mL}$ amylose resin (New England Biolabs) that retained the $\mathrm{PilB}_{\mathrm{Le} 0708}-\mathrm{MBP}$ protein. The column was washed with $200 \mathrm{~mL}$ of PBS buffer, and subsequently, $30 \mathrm{~mL} 10 \mathrm{mM}$ maltose elution buffer was added into the column for protein elution. The protein concentration was determined using a BCA protein assay kit (Sangon Biotech, Shanghai, China), and the purity was assessed by sodium dodecyl sulfate-polyacrylamide gel electrophoresis (SDS-PAGE). The GST-Clp fusion protein was purified according to our earlier study (Xu et al. 2018).

\section{Bacterial adenylate cyclase two-hybrid (BACTH) assay}

The BACTH system was used to test the direct interactions of two proteins of interest at the E. coli background (Ouellette et al. 2017). In brief, the coding region of PilZ $\mathrm{Le}_{\mathrm{L} 639}$ was cloned into $\mathrm{pUT} 18 \mathrm{c}$ to generate the fusion protein comprising $\mathrm{PilZ}_{\mathrm{Le} 3639}$ and the $\mathrm{T} 18$ fragment. The coding genes of other test proteins were individually cloned to pKT25 to make each test protein fused with the T25 fragment. Each recombinant pKT25 plasmid along with the pUT18c-PilZ ${ }_{\mathrm{Le} 3639}$ plasmid were co-transformed into the E. coli BTH101 cells. The resulting $E$. coli strains were cultivated on LB agar plates with 5-bromo-4-chloro-3-indolyl $\beta$-d-galactopyranoside (X-gal) at $50 \mu \mathrm{M}$. The physical interaction of T18 and T25-fused proteins was due to heterodimerization. The latter processs of these hybrid proteins resulted in functional complementation between T25 and T18 fragments, which in turn activate the synthesis of cyclic AMP (cAMP). cAMP binds with the transcription factor CRP to stimulate the transcription of several reporter genes, including genes of the lac operons whose product degrade $\mathrm{X}$-gal resulting in a blue colony formation (Ouellette et al. 2017). The LacZ activity ( $\beta$-galactosidase activity) was quantified as described previously (Ouellette et al. 2017).

\section{Microscale thermophoresis, MST}

The affinity of PilZ $\mathrm{Le}_{\text {L639-His }}$ or GST-Clp binding with cdi-GMP was determined by MST, a powerful technique for quantifying the protein-ligand and protein-protein interactions, using Monolith NT.115 (NanoTemper Technologies, Germany) as described previously (Xu et al. 2018; Han et al. 2020). In brief, the purified GST-Clp or PilZ $_{\mathrm{Le} 3639}-\mathrm{His}_{6}$ was labeled with the fluorescent dye NT647-NHS (Nano Temper Technologies) via amine conjugation. Constant concentration $(500 \mu \mathrm{M})$ of the labeled target protein in a standard MST buffer $(50 \mathrm{mM}$ Tris, $\mathrm{pH}$ 7.5, $150 \mathrm{mM} \mathrm{NaCl}, 10 \mathrm{mM} \mathrm{MgCl} 2,0.05 \%$ Tween 20) was titrated against increasing concentrations of c-di-GMP, which were dissolved in diethylpyrocarbonate-treated water. The MST premium-coated capillaries (Monolith NT.115 MO-K005, Germany) were used to load the samples into the MST instrument at $25^{\circ} \mathrm{C}$ using $80 \%$ MST power, and 20\% LED power. FNorm was plotted on a linear $y$-axis in per mil (\%) against the total concentration of the titrated partner on a $\log _{10} \mathrm{x}$-axis, as reported earlier (Seidel et al. 2013). The experiment was performed in triplicate. Data were analyzed using Nanotemper Analysis software 2.2.4.4577 (NanoTemper Technologies).

\section{Supplementary information}

Supplementary information accompanies this paper at https://doi.org/10. 1186/s42483-020-00054-X.

Additional file 1: Table S1. Strains and plasmids used in this study.

Table S2. Primers used in this study.

Abbreviations

BACTH: Bacterial adenylate cyclase two-hybrid system; c-di-GMP: Bis-(3'-5')cyclic diguanosine monophosphate; HSAF: Heat-stable antifungal factor; MST: Microscale thermophoresis; T4P: Type IV pilus

\section{Acknowledgments}

Not applicable.

\section{Authors' contributions}

GQ conceived the project. GQ and SH designed experiments. LL, MZ carried out experiments. $L L, M Z, D S, S H$, and $G Q$ analyzed data and prepared figures and Tables. LL and GQ wrote the manuscript draft. GQ, AMF, and SHC revised the manuscript. All authors read and approved the final manuscript.

\section{Funding}

This study was supported by the Natural Science Foundation of Jiangsu Province (BK20190026; BK20181325), the National Natural Science Foundation of China (31872016), the Fundamental Research Funds for the Central Universities (KJJQ202001; KYT201805, KYTZ201403 and KYT202001), Jiangsu Agricultural Sciences and Technology Innovation Fund [CX (18)1003] and Innovation Team Program for Jiangsu Universities (2017).

Availability of data and materials

Not applicable.

Ethics approval and consent to participate

Not applicable.

Consent for publication

Not applicable.

Competing interests

The authors have no conflicts of interest to declare.

\section{Author details}

${ }^{1}$ Key Laboratory of Integrated Management of Crop Diseases and Pests, College of Plant Protection, Laboratory of Plant Immunity, Nanjing Agricultural University, No.1 Weigang, Nanjing City 210095, Jiangsu Province, China. ${ }^{2}$ College of Life Science and Technology, Huazhong Agricultural University, Wuhan 430070, China.

Received: 23 March 2020 Accepted: 5 May 2020

Published online: 20 May 2020

\section{References}

Alm RA, Bodero AJ, Free PD, Mttick JS. Identification of a novel gene, pilZ, essential for type 4 fimbrial biogenesis in Pseudomonas aeruginosa. J Bacteriol. 1996;178:46-53. 
Burrows LL. Pseudomonas aeruginosa twitching motility: type IV pili in action. Annu Rev Microbiol. 2012;66:493-520.

Carbonnelle E, Helaine S, Prouvensier L, Nassif X, Pelicic V. Type IV pilus biogenesis in Neisseria meningitidis: PilW is involved in a step occurring after pilus assembly, essential for fibre stability and function. Mol Microbiol. 2005;5: $54-64$.

Chang Y-W, Rettberg LA, Treuner-Lange A, Iwasa J, Søgaard-Andersen L, Jensen GJ. Architecture of the type IVa pilus machine. Science. 2016;351:aad2001.

Chen J, Shen D, Odhiambo BO, Xu D, Han S, Chou S-H, et al. Two direct gene targets contribute to Clp-dependent regulation of type IV pilus-mediated twitching motility in Lysobacter enzymogenes $\mathrm{OH} 11$. Appl Microbiol Biotechnol. 2018;102(17):7509-19.

Chen Y, Xia J, Su Z, Xu G, Gomelsky M, Qian G, et al. Lysobacter PilR, the regulator of type IV pilus synthesis, controls antifungal antibiotic production via a c-diGMP pathway. Appl Environ Microbiol. 2017:83(7):e03397-16.

Chiang P, Sampaleanu LM, Ayers M, Pahuta M, Howell PL, Burrows LL. Functional role of conserved residues in the characteristic secretion NTPase motifs of the Pseudomonas aeruginosa type IV pilus motor proteins PilB, PilT and PilU. Microbiology. 2008;154:114-26.

Christensen P, Cook FD. Lysobacter, a new genus of nonfruiting, gliding bacteria with a high base ratio. Int J Syst Evol Microbiol. 1978;28:367-93.

Corral J, Sebastià P, Coll NS, Barbé J, Aranda J, Valls M. Twitching and swimming motility play a role in Ralstonia solanacearum pathogenicity. mSphere. 2020; 5(2): $: 00740-19$

Craig L, Forest KT, Maier B. Type IV pili: dynamics, biophysics and functional consequences. Nat Rev Microbiol. 2019;17:429-40.

de Bruijn I, Cheng X, de Jager V, Expósito RG, Watrous J, Patel N, et al. Comparative genomics and metabolic profiling of the genus Lysobacter. BMC Genomics. 2015:16:991.

Dunger G, lontop E, Guzzo CR, Farah CS. The Xanthomonas type IV pilus. Curr Opin Microbiol. 2016;30:88-97.

Feng T, Han Y, Li B, Li Z, Yu Y, Sun Q, et al. Interspecies and Intraspecies Signals Synergistically Regulate Lysobacter enzymogenes Twitching Motility. Appl Environ Microbiol. 2019;85(23). https://doi.org/10.1128/AEM.01742-19.

Galperin MY, Chou SH. Structural conservation and diversity of PilZ-related domains. J Bacteriol. 2020;202(4):e00664-19.

Guzzo CR, Salinas RK, Andrade MO, Farah CS. PilZ protein structure and interactions with PilB and the FimX EAL domain: implications for control of type IV pilus biogenesis. J Mol Biol. 2009:393:848-66.

Han S, Shen D, Wang Y-C, Chou S-H, Gomelsky M, Gao Y-G, et al. A YajQ-LysRlike, cyclic di-GMP-dependent system regulating biosynthesis of an antifungal antibiotic in a crop-protecting bacterium, Lysobacter enzymogenes. Mol Plant Pathol. 2020;21(2):218-29.

Hayward AC, Fegan N, Fegan M, Stirling GR. Stenotrophomonas and Lysobacter: ubiquitous plant-associated gamma-proteobacteria of developing significance in applied microbiology. J Appl Microbiol. 2010;108:756-70.

Huang B, Whitchurch CB, Mattick JS. FimX, a multidomain protein connecting environmental signals to twitching motility in Pseudomonas aeruginosa. J Bacteriol. 2003;185:7068-76.

Jain R, Sliusarenko O, Kazmierczak BI. Interaction of the cyclic-di-GMP binding protein FimX and the type 4 pilus assembly ATPase promotes pilus assembly. PLoS Pathog. 2017;13(8):e1006594.

Kobayashi DY, Reedy RM, Palumbo JD, Zhou J-M, Yuen GY. A clp gene homologue belonging to the Crp gene family globally regulates Iytic enzyme production, antimicrobial activity, and biological control activity expressed by Lysobacter enzymogenes strain C3. Appl Environ Microbiol. 2005; 71:261-9.

Li T-N, Chin K-H, Fung K-M, Yang M-T, Wang AH-J, Chou S-H. A novel tetrameric PilZ domain structure from Xanthomonads. PLoS One. 2011;6:e22036.

Li T-N, Chin K-H, Liu J-H, Wang AH-J, Chou S-H. XC1028 from Xanthamanas campestris adopts a PilZ domain-like structure without a c-di-GMP switch. Proteins. 2009;75:282-8.

McCarthy Y, Ryan RP, O'donovan K, He Y-Q, Jiang B-L, Feng J-X, et al. The role of PilZ domain proteins in the virulence of Xanthomonas campestris pv. Campestris. Mol Plant Pathol. 2008;9:819-24.

Ouellette SP, Karimova G, Davi M, Ladant D. Analysis of membrane protein interactions with a bacterial adenylate cyclase-based two-hybrid (BACTH) technique. Curr Protoc Mol Biol. 2017;1 18:20.12.1-20.12.24

Panthee S, Hamamoto H, Paudel A, Sekimizu K. Lysobacter species: a potential source of novel antibiotics. Arch Microbiol. 2016:198:839-45.
Patel N, Cornejo M, Lambert D, Craig A, Hillman BI, Kobayashi DY. A multifunctional role for the type IV pilus in the bacterial biological control agent Lysobacter enzymogenes (abstract). Phytopathology. 2011;101(6):S138.

Patel N, Hillman B, Kobayashi D. Characterization of type IV pilus in the bacterial biocontrol agent Lysobacter enzymogenes strain C3 (abstract). Phytopathology. 2010;100(6):S98.

Qi Y, Xu L, Dong X, Yau YH, Ho CL, Koh SL, et al. Functional divergence of FimX in PilZ binding and type IV pilus regulation. J Bacteriol. 2012;194(21):5922-31.

Qian G, Hu B, Jiang Y, Liu F. Identification and characterization of Lysobacter enzymogenes as a biological control agent against some fungal pathogens. Agric Sci China. 2009;8(1):68-75.

Qian G, Wang Y, Liu Y, Xu F, He Y-W, Du L, et al. Lysobacter enzymogenes uses two distinct cell-cell signaling systems for differential regulation of secondary-metabolite biosynthesis and colony morphology. Appl Environ Microbiol. 2013;79(21):6604-16.

Qian G, Wang Y, Qian D, Fan J, Hu B, Liu F. Selection of available suicide vectors for gene mutagenesis using chiA (a chitinase encoding gene) as a new reporter and primary functional analysis of chiA in Lysobacter enzymogenes strain $\mathrm{OH} 11$. World J Microbiol Biotechnol. 2012;28(2):549-57.

Römling U, Galperin MY, Gomelsky M. Cyclic di-GMP: the first 25 years of a universal bacterial second messenger. Microbiol Mol Biol Rev. 2013;77(1):1-52.

Römling U, Gomelsky M, Galperin MY. C-di-GMP: the dawning of a novel bacterial signaling system. Mol Microbiol. 2005;57:629-39.

Seidel SA, Dijkman PM, Lea WA, van den Bogaart G, Jerabek-Willemsen M, Lazic A, et al. Microscale thermophoresis quantifies biomolecular interactions under previously challenging conditions. Methods. 2013;59(3):301-15.

Xia J, Chen J, Chen Y, Qian G, Liu F. Type IV pilus biogenesis genes and their roles in biofilm formation in the biological control agent Lysobacter enzymogenes OH11. Appl Microbiol Biotechnol. 2018;102:833-46.

Xie Y, Wright S, Shen Y, Du L. Bioactive natural products from Lysobacter. Nat Prod Rep. 2012;29:1277-87.

Xu G, Han S, Huo C, Chin K-H, Chou S-H, Gomelsky M, et al. Signaling specificity in the c-di-GMP-dependent network regulating antibiotic synthesis in Lysobacter. Nucleic Acids Res. 2018;46(18):9276-88.

Yu F, Zaleta-Rivera K, Zhu X, Huffman J, Millet JC, Harris SD, et al. Structure and biosynthesis of heat-stable antifungal factor (HSAF), a broad-spectrum antimycotic with a novel mode of action. Antimicrob Agents Chemother. 2007;51:64-72.

Zhao Y, Qian G, Chen Y, Du L, Liu F. Transcriptional and antagonistic responses of biocontrol strain Lysobacter enzymogenes $\mathrm{OH} 11$ to the plant pathogenic oomycete Pythium aphanidermatum. Front Microbiol. 2017;8:1025.

Zhou X, Qian G, Chen Y, Du L, Liu F, Yuen GY. PilG is involved in the regulation of twitching motility and antifungal antibiotic biosynthesis in the biological control agent Lysobacter enzymogenes. Phytopathology. 2015;105:1318-24.

\section{Ready to submit your research? Choose BMC and benefit from:}

- fast, convenient online submission

- thorough peer review by experienced researchers in your field

- rapid publication on acceptance

- support for research data, including large and complex data types

- gold Open Access which fosters wider collaboration and increased citations

- maximum visibility for your research: over $100 \mathrm{M}$ website views per year

At BMC, research is always in progress.

Learn more biomedcentral.com/submissions 